\title{
Cast shadow can modulate the judged final position of a moving target
}

\author{
SHUICHIRo TAYA \\ University of Surrey, Guildford, England \\ AND \\ KAYO MiURA \\ Kyushu University, Fukuoka, Japan
}

\begin{abstract}
Observers tend to localize the final position of a suddenly vanished moving target farther along in the direction of the target motion (representational momentum). We report here that such localization errors are mediated by perceived motion rather than by retinal motion. By manipulating the cast shadow of a moving target, we induced illusory motion to a target stimulus while keeping the retinal motion constant. Participants indicated the vanishing point of the target by directing a mouse cursor. The resulting magnitude of localization errors was modulated on the basis of the induced direction of the target. Such systematic localization biases were not obtained in a control condition in which the motion paths of the ball and shadow were switched. Our results suggest that cues to object motion trajectory, such as cast shadows, are used for the localization task, supporting a view that a predictive mechanism is responsible for the production of localization errors.
\end{abstract}

When a moving target suddenly vanishes, the judged final position of the target tends to be displaced in the direction of its motion. This localization error was first reported by Freyd and Finke (1984) and was termed representational momentum. Subsequent studies have reported that a number of variables can modulate the displacement. For example, the magnitude of the displacement increases with increased velocity (Freyd \& Finke, 1985; Hubbard \& Bharucha, 1988) and with acceleration (Finke, Freyd, \& Shyi, 1986) of the target. Furthermore, in a manner analogous to physical gravity, the judged final position of a horizontally moving target tends to displace not only forward but also downward (Hubbard, 1990; 1997; Hubbard \& Bharucha, 1988; Nagai, Kazai, \& Yagi, 2002). Friction and centripetal-force-like effects have also been reported (Hubbard, 1995a, 1995b; Nagai \& Yagi, 2001). These similarities between the nature of the displacement and physical principles have supported the conventional view that physical principles that are internalized in the human cognitive system distort the memorized target location (see Hubbard, 2005, for an overview). However, more recent studies have revealed that pursuit eye movements and motion smoothness of the target stimulus have a significant impact on the production of displacement (Kerzel, 2000, 2003b; Kerzel, Jordan, \& Müsseler, 2001). These results suggest that a low-level process - which, in the conventional view, occurs earlier than the cognitive stage - might be involved in this mislocalization phenomenon.
Apart from the controversy about the processing level, it is generally agreed that the localization errors might reflect a function of some predictive mechanism, the role of which is neural delay compensation (Hubbard, 2005, 2006; Kerzel, 2005). The visual system does not have access to on-time information about dynamic environment, because moving objects change their position while signals are transmitted from the retina to the visual cortex. To compensate for the neural delay, the visual system may calibrate the registered target position by extrapolating its past motion trajectory. Consequently, the localization of a suddenly vanishing moving object is displaced in the direction of its motion.

If such a compensation mechanism actually plays a role, we would expect the localization errors to be modulated by visual cues that are useful for the extrapolation process. If so, the subjective or apparent target motion induced by those cues could supersede the objective or retinal target motion in the localization task. Consistent with this notion, previous studies have reported that nonphysical components, such as context (Hubbard, 1993) and observer's anticipation of motion direction (Hubbard, 1994; Verfaillie \& d'Ydewalle, 1991), can modulate the magnitude of localization errors. Those studies suggested that subjective motion can indeed override objective motion (however, see also Nagai \& Saiki, 2005). However, the influence of visual cues on the production of localization errors remains unclear. There are various visual cues in our environment, and we utilize them to achieve more

S. Taya, s.taya@surrey.ac.uk 
reliable perception. If the displacement errors originate from the functional role of our cognitive system, those cues should be implemented in the localization system.

Among the candidates of such visual cues, cast shadows might be the most important, we presumed. First, because they are powerful cues to perceive object motion trajectory (see, e.g., Kersten, Mamassian, \& Knill, 1997; Mamassian, Knill, \& Kersten, 1998), they are quite likely to be helpful in extrapolating the target's motion trajectory. Second, because cast shadows are ubiquitous in the environment, the visual system has plenty of opportunities to develop a mechanism that utilizes shadows for the sake of target localization. In addition, cast shadows are actually an ideal subject for our interests from a practical point of view, because by manipulating shadow-motion paths we can separate the apparent and retinal trajectories of a moving target while keeping retinal motion constant (Kersten et al., 1997); this has enabled us to examine the role of apparent motion in localization by simply testing whether the measured displacement depends on retinal motion or on shadow-induced illusory motion. We tested two predictions about the effects of cast shadows on localization errors by asking observers to set a mouse cursor at the final position of a target. The overview of our stimuli and the rationale of the present study follow.

Figures 1A-1C show example stimuli from our main condition, the valid shadow (VS) condition. We simulated a situation in which a ball is moving while its shadow is cast on a flat floor. Three types of stimulus display were produced by manipulating the shadow's motion path, and they were labeled according to the configurations formed by the motion paths of the ball and shadow. There was a converge display, in which the shadow moved obliquely upward (Figure 1A); a parallel display, in which the shadow moved horizontally (Figure 1B); and a diverge display, in which the shadow moved obliquely downward (Figure 1C). The arrangements of the ball and the shadow could suggest one of three following scenarios: (1) that the ball was descending toward the floor, and receding away from the observer (converge display); (2) that the ball was moving horizontally across the floor (parallel display); or (3) that it was ascending from the floor and approaching the observer (diverge display). In all three stimuli, however, the ball was actually moving only horizontally.

The first goal of the present study was to test whether the shadow-induced approaching and receding motion of the target could modulate the magnitude of the displacement. In particular, we tested the prediction that the diverge display would produce larger forward displacement than either the parallel display or the converge display, according to the illusory motion direction induced by shadows. Note that the cast shadow induced approaching motion in the diverge display (Figure 1C), whereas it induced receding motion in the converge display (Figure 1A). Previous
A
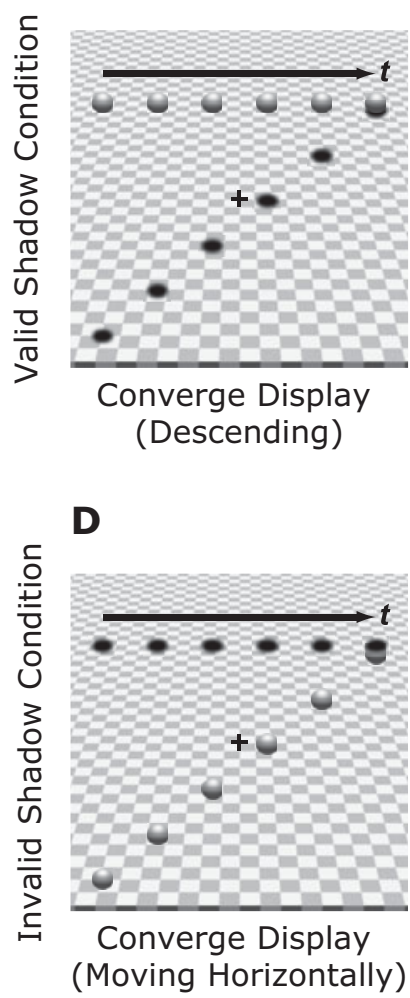

B

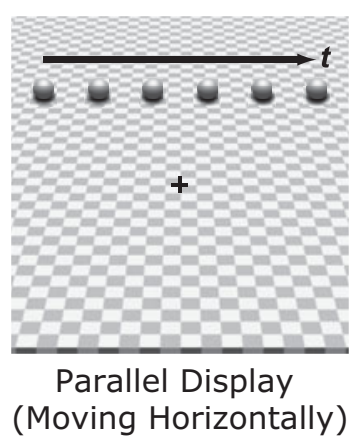

E

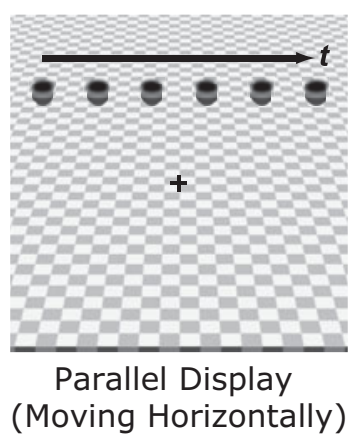

C

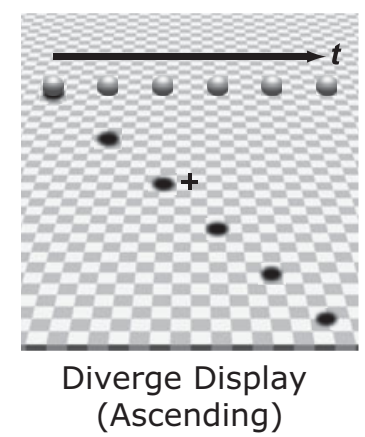

$\mathbf{F}$

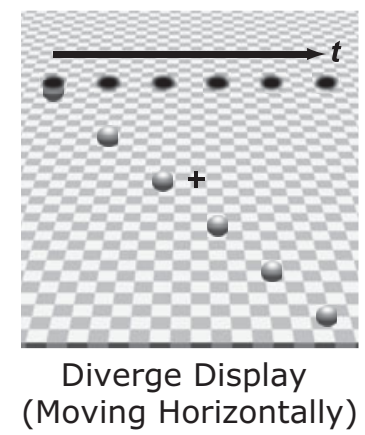

Figure 1. Examples of stimulus displays. The top row (A-C) shows the three test displays in the valid shadow (VS) condition; the bottom row (D-F) shows the displays in the invalid shadow (IS) condition. The words in parentheses underneath each panel describe the expected apparent motion of the target (i.e., balls in the VS condition and shadows in the IS condition). 
studies have reported that the human cognitive system has anisotropic sensitivity to approaching or receding motion that is defined by expanding or contracting motion (Shirai \& Yamaguchi, 2004; Takeuchi, 1997) and by a cast shadow's motion (Imura, Shirai, Tomonaga, Yamaguchi, \& Yagi, 2008). They showed that approaching motion is easier to detect than receding motion. This anisotropy is reasonable from an ecological point of view: We should avoid or intercept an approaching object appropriately in order to survive a dangerous situation such as a collision. This led us to the prediction that if a predictive mechanism is responsible for the localization errors, the approaching target would also have a greater impact on them. Specifically, we predicted that motion extrapolation would be greater for the approaching target (diverge display). This is because a rough but immediate strategy is required in an urgent situation, such as an impending collision, and so creating a larger margin in the prediction of a target's location in the immediate future might be the simplest and most secure strategy. In other words, the localization mechanism may "overreact" to an approaching target in the interest of safety. If so, the apparent diagonal motion of the ball in the diverge display (e.g., from far left to near right in Figure 1C) would be extrapolated much further than that in the other two displays; therefore, displacement would be enlarged along the diagonal dimension in the simulated space. This elongated displacement consists of an in-depth component as well as a horizontal component, and since we measured the displacement on a 2-D display using a mouse-pointing task, we expected to detect the horizontal component of the elongated displacement (i.e., larger forward displacement) in the diverge display.

The second goal of the present study was to investigate the effect of illusory downward and upward motion induced by the shadows. In particular, we tested the prediction that the converge display would produce larger downward displacement than either the parallel display or the diverge display. Note that downward motion of the target is implied in the converge display (Figure 1A), because the descending ball is approaching the floor (i.e., in the direction of the shadow presented just under the target). In other words, the apparent motion of the target in this display could contain a downward vector component. The opposite scenario can be expected with the ascending ball (diverge display, Figure 1C), which is moving away from the floor (i.e., in the opposite direction from its shadow). In this display, the apparent motion of the target could contain an upward vector component. As mentioned earlier, the judged final position of horizontally moving objects tends to be displaced not only forward but also downward (see, e.g., Hubbard \& Bharucha, 1988). If cast shadows are used by the localization mechanism, these shadowinduced upward and downward motions would modulate the magnitude of downward displacements.

We also tested a control condition in which the position of the ball and that of the shadow in the VS condition were switched (Figures 1D-1F). The effect of cast shadow on apparent motion trajectory depends on the light-from-above constraint (Imura et al., 2006); thus, shadow-induced modulations of displacement would not be observed in this invalid shadow (IS) condition. It has been reported that the memorized position of a target tends to be displaced toward nontarget objects (the landmark attraction effect, Hubbard \& Ruppel, 1999; Kerzel, 2002, 2003a; Uddin, Kawabe, \& Nakamizo, 2005). Therefore, even if a larger downward displacement is observed with a descending target (converge display, Figure 1A), this might be simply because the target is attracted to the shadow (nontarget). The IS condition was included in the present study to exclude such alternative accounts.

\section{METHOD}

\section{Participants}

Twenty-six observers participated in the experiment. All of them had normal or corrected-to-normal visual acuity.

\section{Apparatus}

The stimuli were presented on a 15 -in. monitor (Sharp, LL$\mathrm{T} 1502 \mathrm{~T}$ ) with a pixel resolution of $1,024 \times 768$. A PC (IBM, NetVista A30p) controlled the stimulus presentation and data collection. Participants observed the stimulus at a $40-\mathrm{cm}$ viewing distance. Head motion was restricted using a chinrest.

\section{Stimuli in the Valid Shadow Condition}

Figures $1 \mathrm{~A}-1 \mathrm{C}$ show example stimuli from the VS condition. The target was a ball measuring $1.1^{\circ}$ of visual angle. A blurred ellipse (with a $1.4^{\circ}$ long axis and a $0.9^{\circ}$ short axis) was presented as the ball's shadow. The ball and shadow moved smoothly against a checker-patterned background $\left(17.7^{\circ} \times 17.7^{\circ}\right)$, which simulated a flat and horizontal floor situated under eye height. Noticeable motion smear was not observed. A red fixation cross was presented at the center of the display during the stimulus presentation. In all experimental trials, participants were asked to maintain fixation at the fixation cross. The surround of the stimulus area was black.

The VS condition consisted of three test displays and one control display. The ball always traveled along the same horizontal path: It appeared at $7.2^{\circ}$ to the left (or right) of and $5.1^{\circ}$ above the center of the display, and it moved rightward (or leftward) at a velocity of $14.4 \% \mathrm{sec}$. The shadow moved in line with the ball along the vertical axis, taking one of three (i.e., obliquely upward, horizontal, or obliquely downward) different paths, resulting in the three test displays. The velocity of the shadow was $20 \% \mathrm{sec}$ when it moved obliquely and $14.4^{\circ} / \mathrm{sec}$ when it moved horizontally. In the converge display (Figure 1A), the shadow appeared at $11.9^{\circ}$ below the ball and moved obliquely upward; in the parallel display (Figure 1B), the shadow appeared at $0.42^{\circ}$ below the ball and moved parallel to the ball; in the diverge display (Figure 1C), the shadow appeared at $0.42^{\circ}$ below the ball and moved obliquely downward. In the control display, the ball was presented alone, without a shadow.

\section{Stimuli in the Invalid Shadow Condition}

In the IS condition, the motion paths of the ball and shadow in the VS condition were switched (Figure 1D-1F). The target was a blurred ellipse, which was presented as the ball's shadow in the VS condition. The blurred ellipse always moved on a horizontal path, whereas the ball moved on one of three (i.e., obliquely upward, horizontal, or obliquely downward) paths. The resulting three displays were analogous to the three test displays in the VS condition. In the control display, the blurred ellipse was presented alone, without the ball.

\section{Procedure}

In each trial, pressing the space bar initiated the stimulus presentation. The whole stimulus display with fixation cross was initially presented statically for $1,000 \mathrm{msec}$ to ensure fixation. Then, the ball and the shadow moved and disappeared 200, 400, 600, 800, or 1,000 msec later (corresponding to the target's travel distances 
of $2.88^{\circ}, 5.76^{\circ}, 8.64^{\circ}, 11.52^{\circ}$, and $14.4^{\circ}$, respectively). The stimulus turned black at the end of the motion. Immediately after the stimulus disappearance, a $1.1^{\circ} \times 1.1^{\circ}$ crosshair mouse cursor was presented with a $17.7^{\circ} \times 17.7^{\circ}$ white outlined square (to reference the stimulus area). The initial position of the cursor was randomized within a circular region of $4.3^{\circ}$ radius centered on the true final position of the target. The participants' task was to point the cursor as accurately as possible at the final position of the target. No feedback was given.

All stimuli appeared twice in random order in each of the two conditions. Therefore, each condition consisted of 80 trials incorporating five durations $(200,400,600,800$, and $1,000 \mathrm{msec})$, two motion directions (leftward or rightward), four types of stimulus display (parallel, converge, diverge, and control), and two repetitions. The participants completed 10 practice trials before the main experiment. Half of the participants performed the VS condition first.

After all trials were completed, the participants were shown example stimuli and were asked what kind of target motion they had observed. Only 1 participant reported an inability to obtain an impression of ascending or descending motion of the ball in the VS condition. The data of the exceptional participant were excluded from later analyses.

\section{RESULTS}

\section{Magnitude of Displacements}

We evaluated the magnitude of displacement by subtracting the indicated final position of the target from the actual final position. We refer here to the calculated value in the horizontal axis as forward displacement and to that in the vertical axis as downward displacement.

Tables 1 and 2 show the average forward and downward displacement, respectively $(N=25)$. The average and standard errors presented here were calculated after collapsing across five durations, two motion directions, and two repetitions. The positive values in Table 1 indicate that the judged final position was displaced forward along the direction of the target's motion. The positive values in Table 2 indicate that the judged final position was displaced down-
Table 1

Magnitude of Forward Displacement (in Degrees of Visual Angle)

\begin{tabular}{lccccc}
\hline & \multicolumn{3}{c}{ Shadow Validity } \\
\cline { 2 - 3 } \cline { 5 - 6 } Display & \multicolumn{2}{c}{ Valid } & & \multicolumn{2}{c}{ Invalid } \\
\cline { 2 - 3 } \cline { 5 - 6 } & $M$ & $S E$ & & $M$ & $S E$ \\
\hline Diverge & 0.53 & 0.09 & & 0.34 & 0.08 \\
Parallel & 0.26 & 0.08 & & 0.23 & 0.08 \\
Converge & 0.33 & 0.07 & & 0.27 & 0.06 \\
Control & 0.23 & 0.08 & & 0.28 & 0.08 \\
\hline
\end{tabular}

Note- $N=25$.

Table 2

Magnitude of Downward Displacement (in Degrees of Visual Angle)

\begin{tabular}{cccccc}
\hline & \multicolumn{4}{c}{ Shadow Validity } \\
\cline { 2 - 3 } \cline { 5 - 6 } Display & \multicolumn{2}{c}{ Valid } & & \multicolumn{2}{c}{ Invalid } \\
\cline { 2 - 3 } \cline { 5 - 6 } & $M$ & $S E$ & & $M$ & $S E$ \\
\hline Diverge & 0.79 & 0.09 & & 0.58 & 0.08 \\
Parallel & 0.91 & 0.08 & & 0.68 & 0.08 \\
Converge & 0.98 & 0.10 & & 0.58 & 0.08 \\
Control & 0.85 & 0.09 & & 0.61 & 0.08 \\
\hline
\end{tabular}

Note $-N=25$.

ward. All observed displacements were different from zero $(p<.05$, one-sample $t$ test with Bonferroni correction).

\section{Modulation of Forward Displacement}

To assess the effect of cast shadows on forward displacement, we subtracted the forward displacement of the control trial from that of each test trial. Figure 2A shows the averaged value after the calculation $(N=25)$. A threeway repeated measures ANOVA with shadow validity (valid, invalid), display type (converge, parallel, diverge), and duration $(200,400,600,800$, and $1,000 \mathrm{msec})$ as fac-
B

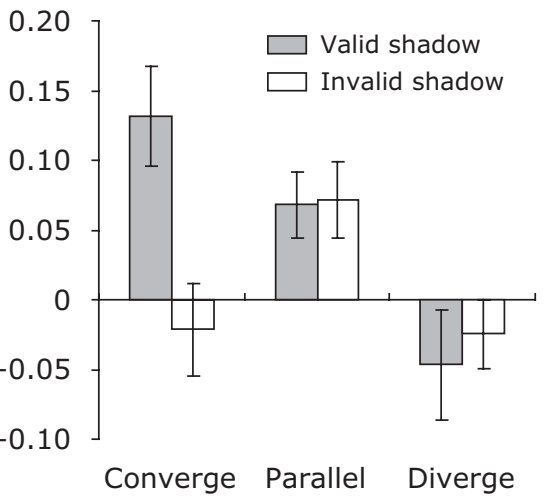

Stimulus Display

Stimulus Display

Figure 2. (A) The modulation of forward displacement. Positive values mean that the forward displacement in each test display was larger than that in the control. (B) The modulation of downward displacement. Positive values mean that the downward displacement in each test display was larger than that in the control. Gray bars show the results of the valid shadow condition; white bars show the results of the invalid shadow condition. Error bars represent 1 standard error of the mean. The averages and standard errors were calculated after collapsing each condition across duration, motion direction, and repetition $(N=25)$. 

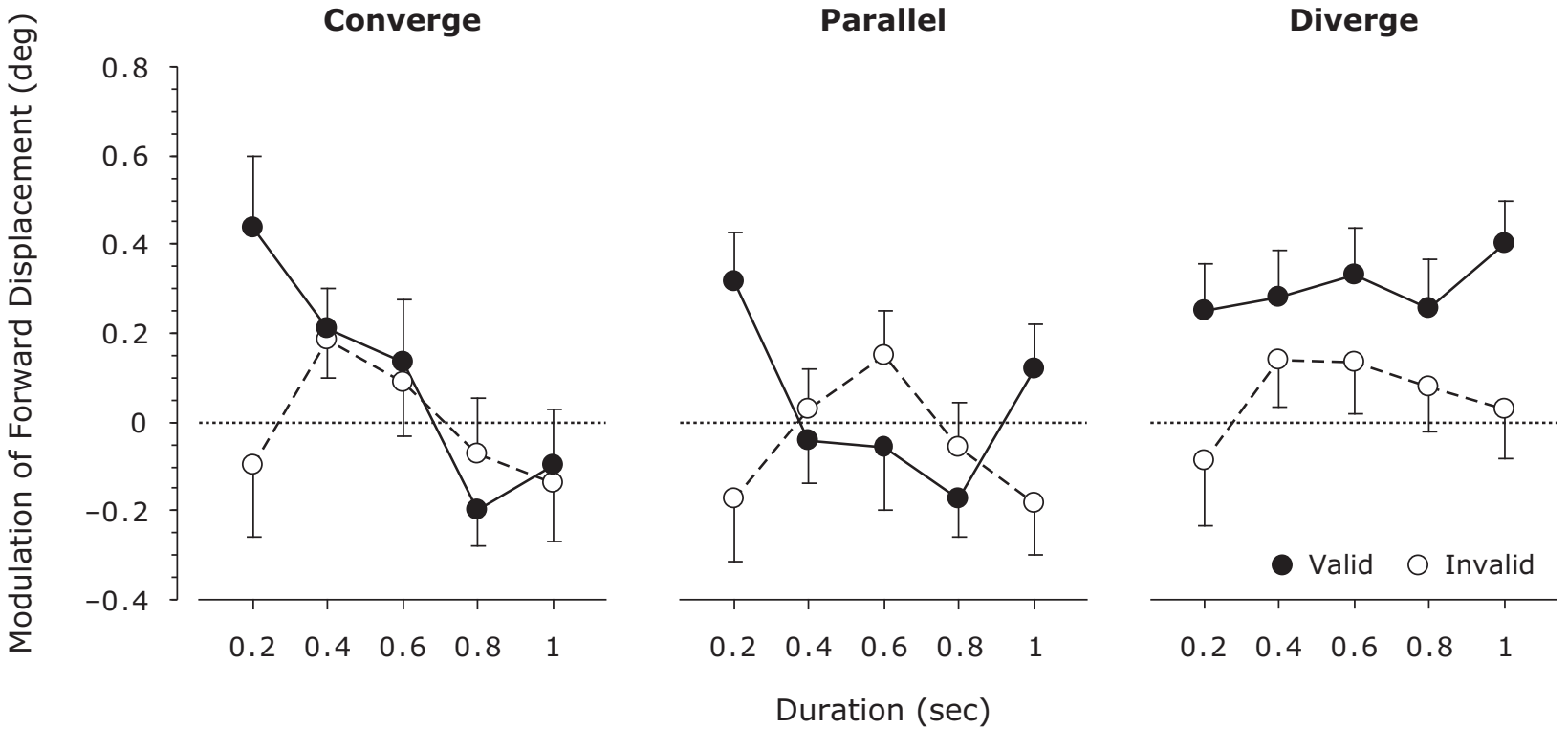

$\begin{array}{lllll}0.2 & 0.4 & 0.6 & 0.8 & 1\end{array}$

Figure 3. The averaged modulation of forward displacements (i.e., difference from the control display), plotted for each of five durations. Error bars denote 1 standard error of the mean. Averages and standard errors were calculated after collapsing the data across motion direction and repetition $(N=25)$.

tors revealed a significant main effect of shadow validity $[F(1,24)=23.13, p<.001]$ and display type $[F(2,48)=$ $11.66, p<.001]$. The main effect of duration was not significant $(p>.05)$. Interactions were significant for all combinations of factors [shadow validity $X$ display type, $F(2,48)=3.78, p<.05$; shadow validity $\times$ duration, $F(4,96)=3.31, p<.05$; display type $\times$ duration, $F(8,192)=4.86, p<.001$; shadow validity $\times$ display type $\times$ duration, $F(8,192)=2.96, p<.005]$. The simple main effect of display type was significant under the VS condition $[F(2,96=14.97, p<.001]$, but not under the IS condition $(p>.1)$. Post hoc comparison with Ryan's (1960) method under the VS condition revealed that the modulation of forward displacement was larger in the diverge display than in the other two test displays (diverge display vs. parallel display, $t=5.24, p<.05$; diverge display vs. converge display, $t=3.98, p<.05$ ). These results suggest that the apparent approaching motion produced the larger displacement, as we predicted.

The significant three-way interaction suggests that the effect of shadow validity depended on display type and duration (Figure 3). To see this point in more detail, the three-way interaction was broken down further via separate two-way ANOVAs, with shadow validity (valid, invalid) and duration $(200,400,600,800,1,000 \mathrm{msec})$ as factors, for each display type. In the diverge display, only the main effect of shadow validity was significant $[F(1,24)=$ $33.94, p<.001$ ], suggesting that the VS condition produced larger displacement than the IS condition, regardless of the duration (see Figure 3, right panel). Interaction between shadow validity and duration was significant, however, in the parallel display $[F(4,96)=5.25, p<.001]$ and in the converge display $[F(4,96)=3.27, p<.05]$. The main effect of duration was also significant in the converge display $[F(4,96)=6.19, p<.001]$, but no other main effects were significant in the converge display or the diverge display. In those displays, as shown in Figure 3, the displacement was larger in the VS condition than in the IS condition only at the shortest or longest duration (i.e., when the judgments were made in peripheral vision). These results suggest that, in peripheral vision, the VS condition produced larger forward displacement even when the shadow would not have had an effect on the ball's apparent motion (i.e., the parallel display). The origin of this peripheral effect might be the lower discriminability of the ball's boundary. In the VS condition, the target (ball) and background (floor) were relatively similar in color compared with those in the IS condition, which could induce a difficulty in discriminating the ball's boundary, resulting in the enhanced magnitude of displacement. ${ }^{1}$

To summarize the findings here, we found a larger modulation of forward displacement in the diverge display of the VS condition, which might have been caused by the shadow-induced approaching motion of the ball. The magnitude of the forward displacement was larger in the diverge display than in the other two test displays under the VS condition, whereas there were no differences among the three test displays under the IS condition. These results are congruent with our prediction that shadow-induced approaching motion would produce larger forward displacement. The VS condition also produced larger forward displacements with the parallel display as well as with the converge display, but only when the measurements were made in peripheral vision. Those results might originate from a residual factor (probably lower discriminability of the ball's boundary).

\section{Modulation of Downward Displacement}

The effect of stimulus type on downward displacement was calculated by subtracting the downward displacement 


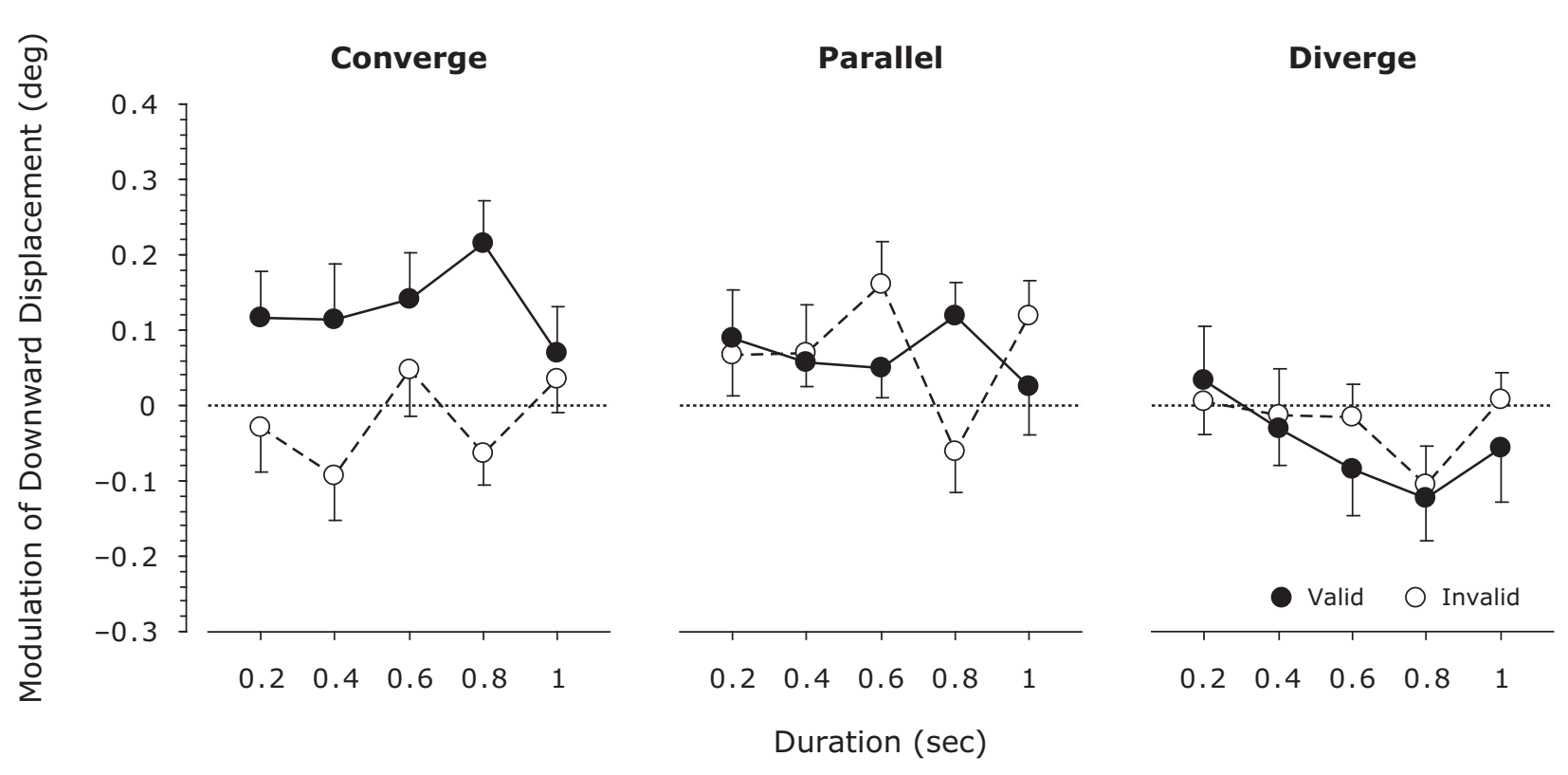

Figure 4. The average modulation of downward displacements (i.e., difference from the control display), plotted for each of five durations. Error bars denote 1 standard error of the mean. Averages and standard errors were calculated after collapsing the data across motion direction and repetition $(N=25)$.

of the control trial from that of each test trial. Figure 2B shows the value after that calculation, averaged over 15 participants, and Figure 4 shows the results of the three displays plotted for each of the five durations. A three-way repeated measures ANOVA with shadow validity (valid, invalid), display type (converge, parallel, diverge), and duration $(200,400,600,800,1,000 \mathrm{msec})$ as factors revealed a significant main effect of display type $[F(2,48)=10.17$, $p<.001]$. More importantly, interaction between shadow validity and display type was significant $[F(2,48)=10.73$, $p<.001]$. No other main effects or interactions were significant. We then tested the simple main effect of shadow validity to see which of the three test displays showed a significant difference between the VS condition and the IS condition. The simple main effect of shadow validity was significant in the converge display $[F(1,72=16.17, p<$ $.001]$ but not in the other test displays $(p>.1)$, indicating that the converge display (descending ball) produced larger downward displacement in the VS condition than in the IS condition.

The larger downward displacements obtained in the converge display were concordant with our prediction that the apparent downward motion would enhance the magnitude of downward displacement. As has been reported elsewhere, the judged final position tends to shift downward even when the target moves horizontally (Hubbard, 1990; 1997; Hubbard \& Bharucha, 1988). Furthermore, downward target motion produces a larger displacement than does upward target motion (Hubbard \& Bharucha, 1988; Nagai et al., 2002). The results of these studies suggest that knowledge about gravitational attraction is implemented in the mechanism responsible for the localization errors. In the converge display of our VS condition, the target was descending toward the floor; thus, the effect of gravity was more obvious in this display than in the other test displays.
The direction of the attractive force of gravity was always downward (i.e., the direction of shadow, which was always presented in line with the ball along the vertical axis), projected onto the computer display in parallel with the vertical axis of the display screen. Participants' mouse settings might have been affected by this downward component of apparent motion, resulting in the larger downward displacement in the converge display of the VS condition.

\section{DISCUSSION}

We demonstrated here that shadow-induced illusory motion can systematically modulate judgments of the final position of a moving target. The goal of this study was to test our prediction that the shadow-induced apparent motion can modulate the magnitude of forward displacement and downward displacement. In accordance with our prediction, the results of the VS condition showed (1) greater forward displacement in the diverge display, in which the cast shadow specified approaching motion of the target, and (2) greater downward displacement in the converge display, in which apparent downward motion of the target was induced by the shadow. These results suggest that cast shadows are used by our visual system as a cue for predicting the future position of moving targets.

The results of the control condition (the IS condition) excluded the alternative explanation that the effect of cast shadows obtained in the VS condition occurred because the memorized position of the target was simply displaced toward its moving shadow (Hubbard \& Ruppel, 1999; Kerzel, 2002, 2003a; Uddin et al., 2005). The retinal motion paths of the target and the nontarget in the IS condition were exactly the same as those in the VS condition. Therefore, if the judged position was simply biased toward the nontarget object, the stimuli of the IS condition would 
have produced displacement patterns similar to those of the VS condition. However, the results of the IS condition were quite different from those of the VS condition. In the IS condition, unlike in the VS condition, the motion of a nontarget (the ball) did not systematically bias the localization of the target (the blurred ellipse).

Our findings agree with the hypothesis that the displacements are the result of a functional mechanism that predicts the future position of a moving target by extrapolating its motion trajectory (Hubbard, 2005; Kerzel, 2005). Note that the shadow-induced illusory motion used here was not delusive but rather a straightforward interpretation of the external world. In the situation simulated in the VS stimuli, the targets showed the same retinal motion across the three test displays; however, they were actually traveling along different motion trajectories in the simulated space. Cast shadows can help us to reveal that these targets actually had different motion directions, even though their trajectories happened to be projected on the same retinal coordination. In this sense, the illusory motion induced by shadows was more helpful than retinal motion for extrapolating the future position of the target. Our results suggest that the mechanism underlying the localization errors may depend on the contextual information provided by cast shadows.

In contrast with our findings, Nagai and Saiki (2005) reported that neither illusory direction nor illusory speed could modulate the magnitude of the displacement. The conflicting results might have been caused by the differences in fixation control. Participants in the previous study were not required to fixate on a fixation point and thus were likely to pursue a moving target with their eyes. In contrast, our participants were asked to maintain fixation throughout a trial. Although pursuit eye movement is not indispensable for the production of displacement (Kerzel, 2003b; Kerzel \& Gegenfurtner, 2003; Müsseler, Stork, \& Kerzel, 2002), it has been suggested that the magnitude of displacement could be greatly reduced, or in some cases go unobserved, with eye fixation (Kerzel, 2000, 2006; Kerzel et al., 2001). Therefore, at least eye motion may strengthen the magnitude of displacement. The pursuit eye movement probably enhanced the displacement in Nagai and Saiki's study, and that might have overridden or masked the effect of illusory motion.

In summary, we demonstrated here that displacements of judged final position of a moving target can be modulated by the illusory motion induced by a target's cast shadow. To our knowledge, this is the first study showing that this type of localization error can be modulated by a visual cue to object motion trajectory. We conclude that the mechanism underlying displacements is sensitive to the motion information provided by cast shadows. Our findings agree with the view that such a mechanism has been developed for predicting the future position of a moving target by extrapolating the target motion trajectory.

\section{AUTHOR NOTE}

This research was supported by Engineering and Physical Sciences Research Council Grant EP/F069421/1 to S.T. and Grant-in-Aid for Scientific Research 20330153 from the Japanese Society for the Promotion of Science to K.M. We thank Daniel Baker, Takahiro Kawabe, Hiroyuki Mitsudo, and Gerard Remijn for comments on an earlier draft of this article. Correspondence concerning this article should be addressed to S. Taya, Department of Psychology, University of Surrey, Guildford, Surrey GU2 7XH, England (e-mail: s.taya@surrey.ac.uk).

\section{REFERENCES}

Finke, R. A., Freyd, J. J., \& Shyi, G. C. (1986). Implied velocity and acceleration induce transformations of visual memory. Journal of Experimental Psychology: General, 115, 175-188. doi:10.1037/0096 $-3445.115 .2 .175$

Freyd, J. J., \& Finke, R. A. (1984). Representational momentum. Journal of Experimental Psychology: Learning, Memory, \& Cognition, 10, 126-132. doi:10.1037/0278-7393.10.1.126

FREYD, J. J., \& Finke, R. A. (1985). A velocity effect for representational momentum. Bulletin of the Psychonomic Society, 23, 443-446.

HubBard, T. L. (1990). Cognitive representation of linear motion: Possible direction and gravity effects in judged displacement. Memory \& Cognition, 18, 299-309.

HubBARD, T. L. (1993). The effect of context on visual representational momentum. Memory \& Cognition, 21, 103-114.

HubBard, T. L. (1994). Judged displacement: A modular process? American Journal of Psychology, 107, 359-373.

HubBard, T. L. (1995a). Cognitive representation of motion: Evidence for friction and gravity analogues. Journal of Experimental Psychology: Learning, Memory, \& Cognition, 21, 241-254. doi:10.1037/0278 $-7393.21 .1 .241$

HubBARD, T. L. (1995b). Environmental invariants in the representation of motion: Implied dynamics and representational momentum, gravity, friction, and centripetal force. Psychonomic Bulletin \& Review, 2, 322-338.

Hubbard, T. L. (1997). Target size and displacement along the axis of implied gravitational attraction: Effects of implied weight and evidence of representational gravity. Journal of Experimental Psychology: Learning, Memory, \& Cognition, 23, 1484-1493. doi:10.1037/0278 -7393.23.6.1484

HubBARD, T. L. (2005). Representational momentum and related displacements in spatial memory: A review of the findings. Psychonomic Bulletin \& Review, 12, 822-851.

HubBard, T. L. (2006). Bridging the gap: Possible roles and contributions of representational momentum. Psicológica, 27, 1-34.

Hubbard, T. L., \& Bharucha, J. J. (1988). Judged displacement in apparent vertical and horizontal motion. Perception \& Psychophysics, 44, 211-221

HubBard, T. L., \& Ruppel, S. E. (1999). Representational momentum and the landmark attraction effect. Canadian Journal of Experimental Psychology, 53, 242-256.

Imura, T., Shirai, N., Tomonaga, M., Yamaguchi, M. K., \& Yagi, A. (2008). Asymmetry in the perception of motion in depth induced by moving cast shadows. Journal of Vision, 8(13, Art. 10), 1-8. doi: $10.1167 / 8.13 .10$

Imura, T., Yamaguchi, M. K., Kanazawa, S., Shirai, N., Otsuka, Y., Tomonaga, M., \& YAGI, A. (2006). Perception of motion trajectory of object from the moving cast shadow in infants. Vision Research, 46, 652-657. doi:10.1016/j.visres.2005.07.028

Kersten, D., Mamassian, P., \& Knill, D. C. (1997). Moving cast shadows induce apparent motion in depth. Perception, 26, 171-192.

KerzeL, D. (2000). Eye movements and visible persistence explain the mislocalization of the final position of a moving target. Vision Research, 40, 3703-3715. doi:10.1016/S0042-6989(00)00226-1

KERZEL, D. (2002). Memory for the position of stationary objects: Disentangling foveal bias and memory averaging. Vision Research, 42, 159-167. doi:10.1016/S0042-6989(01)00274-7

KerzeL, D. (2003a). Attention maintains mental extrapolation of target position: Irrelevant distractors eliminate forward displacement after implied motion. Cognition, 88, 109-131.

KERZEL, D. (2003b). Mental extrapolation of target position is strongest with weak motion signals and motor responses. Vision Research, 43, 2623-2635. doi:10.1016/S0042-6989(03)00466-8

KerzeL, D. (2005). Representational momentum beyond internalized physics: Embodied mechanisms of anticipation cause errors in visual 
short-term memory. Current Directions in Psychological Science, 14, 180-184.

Kerzel, D. (2006). Why eye movements and perceptual factors have to be controlled in studies on "representational momentum." Psychonomic Bulletin \& Review, 13, 166-173.

Kerzel, D., \& Gegenfurtner, K. R. (2003). Neuronal processing delays are compensated in the sensorimotor branch of the visual system, Current Biology, 13, 1975-1978. doi:10.1016/j.cub.2003.10.054

Kerzel, D., Jordan, J. S., \& Müsseler, J. (2001). The role of perception in the mislocalization of the final position of a moving target. Journal of Experimental Psychology: Human Perception \& Performance, 27, 829-840. doi:10.1037/0096-1523.27.4.829

Mamassian, P., Knill, D. C., \& Kersten, D. (1998). The perception of cast shadows. Trends in Cognitive Sciences, 2, 288-295. doi:10.1016/ S1364-6613(98)01204-2

Müsseler, J., Stork, S., \& Kerzel, D. (2002). Comparing mislocalizations with moving stimuli: The Fröhlich effect, the flash-lag, and representational momentum. Visual Cognition, 9, 120-138. doi:10.1080/13506280143000359

NAGAI, M., KAZAI, K., \& YAGI, A. (2002). Larger forward memory displacement in the direction of gravity. Visual Cognition, 9, 28-40. doi:10.1080/13506280143000304

NAGAI, M., \& SAIKI, J. (2005). Illusory motion and representational momentum. Perception \& Psychophysics, 67, 855-866.

NAGAI, M., \& YAGI, A. (2001). The pointedness effect on representational momentum. Memory \& Cognition, 29, 91-99.
RYAN, T. A. (1960). Significance test for multiple comparisons of proportions, variances, and other statistics. Psychological Bulletin, 57, 318-328.

Shirai, N., \& Yamaguchi, M. K. (2004). Asymmetry in the perception of motion-in-depth. Vision Research, 44, 1003-1011. doi:10.1016/ j.visres.2003.07.012

TAKEUCHI, T. (1997). Visual search of expansion and contraction. Vision Research, 37, 2083-2090. doi:10.1016/S0042-6989(96)00225-8

Uddin, M. K., Kawabe, T., \& NaKamizo, S. (2005). Attention shift not memory averaging reduces foveal bias. Vision Research, 45, 33013306. doi:10.1016/j.visres.2005.03.006

Verfaillie, K., \& D’Ydewalle, G. (1991). Representational momentum and event course anticipation in the perception of implied periodical motions. Journal of Experimental Psychology: Learning, Memory, \& Cognition, 17, 302-313. doi:10.1037/0278-7393.17.2.302

\section{NOTE}

1. The peripheral effect was not obvious at the longest duration $(1 \mathrm{sec})$ of the converge display. This is presumably because the ball in this display would be expected to "bounce off" the floor at the longest duration, and this expectation would reduce the displacement, canceling the peripheral effect (see Hubbard \& Bharucha, 1988).

(Manuscript received October 20, 2009; revision accepted for publication May 31, 2010.) 